\title{
Intensified Surveillance for Early Detection of Breast Cancer in High-Risk Patients
}

\author{
Ulrich Bick \\ Department of Radiology, Charité Berlin, Germany
}

\section{Keywords}

Genes, BRCA1 - Genes, BRCA2 - Breast neoplasms .

Early detection of cancer . Magnetic resonance imaging

\section{Summary}

Efforts for early detection of breast cancer play an important role in the care of high-risk women. This will include both women with a pathological mutation in one of the known breast cancer susceptibility genes as well as women with a high breast cancer risk based on family history only. Due to the much higher incidence of breast cancer in premenopausal women with a genetic predisposition or a familial background, to be most effective, imaging-based breast surveillance should start at an age as early as $25-30$ years. There is now ample evidence that magnetic resonance imaging (MRI) is by far the most sensitive imaging modality in young high-risk women. With high-risk multimodality screening at least $30 \%$ of breast cancers will be detected primarily by MRI and would have been missed at regular screening without MRI. Therefore, most high-risk breast surveillance programs now offer annual MRI to eligible high-risk women from age 25 to 30 , usually supplemented by regular mammography starting at least from age 40 . The inclusion of clinical breast exam (CBE) and/or ultrasound in the high-risk surveillance has little impact on the detection of additional cancers, but may improve compliance and reduce unnecessary callbacks for nonspecific findings on MRI. To reduce advanced stage interval cancers, especially in BRCA1/2 mutation carriers, some programs offer additional semiannual CBE and/or ultrasound or alternate MRI and mammography every 6 months. How long regular MRI should be continued in high-risk women is a matter of considerable debate. It appears feasible that MRI can safely be discontinued even in BRCA1/2 mutation carriers between the age of 60 and 70, especially if mammographic breast density is low. Even though several cohort studies have now demonstrated a very favorable stage distribution of breast cancers found in women undergoing high-risk surveillance with MRI, data on long-term survival and mortality in these patients is still rare.

\section{Introduction}

Women with a genetic predisposition for breast cancer develop breast cancer more frequently and at an earlier age than women without such a predisposition. Without risk-reducing strategies such as mastectomy or salpingo-oophorectomy, the cumulative risk for developing breast cancer up to the age of 70 is around $60 \%$ for BRCA1 and 55\% for BRCA2 mutation carriers [1]. As more than half of the of the breast cancers in BRCA1/2 carriers occur before the age of 50 [1], the typical strategies for early detection of breast cancer in the general population, such as organized mammography screening starting at age 50, are not adequate for these high-risk women. Since the sensitivity of mammography in premenopausal women with high breast parenchymal density is reduced [2], and risks associated with the radiation exposure from mammography are higher in younger women [3], alternative imaging modalities such as breast ultrasound or magnetic resonance imaging (MRI) of the breast should play a more prominent role in screening young women with a genetic predisposition. Therefore, many countries have recently implemented intensified multimodality surveillance programs that include breast MRI for high-risk women [4-6]. This paper reviews and discusses the available evidence regarding the risks and benefits of such screening strategies.

\section{Selecting Women for Intensified Surveillance}

There is now general agreement that women with a proven pathological mutation in one of the high penetrance breast cancer

\section{KARGER \\ Fax +497614520714

\section{() 2015 S. Karger GmbH, Freiburg}

$1661-3791 / 15 / 0101-0013 \$ 39.50 / 0$
Prof. Dr. Ulrich Bick 
susceptibility genes such as BRCA1 and BRCA2 should be offered additional intensified surveillance strategies beyond regular mammography screening. However, even among BRCA1/2 carriers, individual breast cancer risk will vary substantially based on the existence of genetic modifiers in the family [1] as well as lifestyle factors [7]. When selecting women for high-risk surveillance, it is also important to have strategies in place to deal with rare variants of uncertain significance (VUS) in the BRCA1/2 genes [8].

Appropriate recommendations for women with a strong family history of breast cancer but no known mutation in one of the high penetrance genes are much more difficult to establish. Usually, in women with an unknown mutation status, a threshold based on estimated lifetime risk or carrier probability is used to select women for intensified surveillance. The National Institute for Health and Care Excellence (NICE) in the UK recommends annual screening with MRI starting at age 30 for women with a BRCA $1 / 2$ carrier risk of $30 \%$ or more [9]. The American Cancer Society (ACS) recommends screening with MRI in addition to mammography for untested women with a first-degree relative with a known BRCA $1 / 2$ mutation or for women with a lifetime risk of $20-25 \%$ or greater based on family history [10]. The German Consortium of Hereditary Breast and Ovarian Cancer (GC-HBOC) offers its intensified multimodal surveillance program to women without a mutation in one of the known high or moderate penetrance genes, if the remaining breast cancer lifetime risk is at least $30 \%$ and/or if the BRCA1/2 carrier probability is at least $20 \%$ [11]. In the GCHBOC, the software program Cyrillic 2.1 (http://www.cyrillicsoftware.com/) is currently still used for the risk calculation, but the results of the risk calculation may vary substantially depending on the algorithm used [12].

Since the lifetime risk of contralateral breast cancer can be as high as $83 \%$ for BRCA1 and $62 \%$ for BRCA 2 mutation carriers [1], most programs also offer the intensified surveillance to woman with a personal history of breast cancer if they fulfill the specified risk criteria for entry into the program [5].

Another group of women with a significantly elevated risk of breast cancer before the age of 50, similar to BRCA1/2 carriers, are women who received thoracic mantle field radiotherapy for Hodgkin's disease during childhood or adolescence. Therefore, it is usually recommended that these women should be offered the same intensified surveillance, including MRI, as other high-risk patients $[5,13]$.

\section{Duration and Frequency of High-Risk Screening}

Based on the available data on age-specific breast cancer incidence in BRCA1/2 carriers $[1,14]$, intensified surveillance including annual MRI should be offered to high-risk women starting at least from age 30 [9]. Since a small number of BRCA1/2 carriers develop breast cancer even before age 30 and these cancers are often of high clinical relevance, the GC-HBOC offers screening to women with a known BRCA1/2 mutation already from age 25 (table 1). Very early onset of breast cancer in a family member (e.g. before the age of 30) may be considered a reason to start screening even earlier, with the usual recommendation to start screening at least 5 years before the earliest breast cancer diagnosis in the family. Another group of patients in whom breast cancer screening may have to start as early as age 20-25 years are women with TP53 mutations (Li-Fraumeni syndrome) [9, 15]. Most recommendations agree that the intensified surveillance, including annual MRI, should be continued at least until the age of 50 [16], when most regular mammography screening programs for the general population start. However, the relative incidence rates for breast cancer for BRCA1/2 carriers older than 50 remain high (at least 10 times higher) compared to the general population [14], and since the additional benefit in breast cancer detection of MRI over mammography is relatively independent of age [17], it appears advisable to continue the intensified surveillance with annual MRI in BRCA1/2 mutation carriers until breast parenchymal density is sufficiently low to allow for optimal imaging conditions with mammography, but usually not beyond age 70 [5].

Intensified surveillance is also no longer necessary after bilateral therapeutic or prophylactic mastectomy, as long as the breast parenchyma has been adequately removed during the procedure [5]. If necessary, this can easily be confirmed by MRI following the surgery [18].

Whether an annual screening frequency is sufficient in BRCA1/2 mutation carriers is a matter of considerable debate. Some cancers, especially in BRCA1-carriers, present as palpable interval cancers, usually between 6 and 12 months after a normal annual screening exam with MRI $[17,19]$. Some high-risk screening programs therefore include semiannual clinical breast exam (CBE) and/or breast ultrasound in addition to the regular annual screening round with MRI $[11,20,21]$ or alternate MRI and mammography every 6 months [22]

\section{Breast MRI}

MRI of the breast is by far the most sensitive imaging modality for the detection of both invasive and in situ breast cancer, and represents the cornerstone of any high-risk surveillance program for breast cancer. Even if used alongside CBE, ultrasound, and mammography in a high-risk multimodality screening program, at least $30 \%$ of cancers will be detected primarily by MRI and would have been missed at regular screening without MRI $[4,5]$. If measured against all cancers detected at multimodality screening or during the 12-month interval following the particular screening round, MRI has a sensitivity of $\sim 90 \%$ compared to not more than $60 \%$ for mammography and ultrasound combined [23]. Cancers missed at MRI in this calculation include cancers detected incidentally at prophylactic surgery, true clinical interval cancers predominantly in BRCA1 carriers and a small number of cancers associated with microcalcifications detected solely by mammography (mostly non-high-grade ductal carcinoma in situ (DCIS)). The sensitivity of MRI especially in regards to DCIS is strongly dependent on image quality and reader experience [24, 25]. Modern high-field (1.5 $\mathrm{T}$ or higher) magnets coupled with dedicated multichannel 
breast coils enable combined high temporal ( 1 min or less per dynamic sequence) and high spatial resolution $(<1 \mathrm{~mm}$ in plane resolution) imaging, which allows detection of cancers as small as 3-5 mm. For optimal results, appropriate quality assurance measures similar to organized mammography screening should be in place for screening with MRI, including minimal requirements for the imaging protocol and reader experience as well as structured double reading $[4,26,27]$.

As many benign abnormalities including fibroadenomas, lymph nodes, fibrocystic changes and pathological high-risk lesions (such lobular neoplasia or flat epithelial atypia) may show enhancement on MRI, false-positive findings are unavoidable in MRI screening and may be as high as $10-15 \%$, especially in the first (prevalence) screening round [5]. The specificity of MRI can be increased by timing the MRI exam in the appropriate menstrual cycle phase (second week) in premenopausal women [28] and by discouraging the use of hormonal contraception or hormone replacement therapy prior to MRI. Also, risk-reducing salpingo-oophorectomy and long-term adjuvant endocrine therapy in high-risk women previously diagnosed with breast cancer will create optimal imaging conditions for MRI.

The addition of mammography and tailored second-look ultrasound can further increase the specificity of MRI, especially for non-mass-like enhancement [29]. However to avoid delay in diagnosis [30], small enhancing mass lesions on MRI, which are new or increased in size, should be biopsied or at least subjected to shortterm follow-up, even if no corresponding correlate is found on mammography or second-look ultrasound. This is especially true for BRCA1 mutation carriers, in whom an otherwise non-descript small enhancing mass lesion may represent an aggressive triplenegative breast cancer (TNBC).

With appropriate safeguards (e.g. single-dose protocols, use of low nephrogenic systemic fibrosis-risk contrast media and screening for renal function impairment), contrast-enhanced MRI is a very safe procedure [31]. However, MRI cannot be performed in a small number of patients, e.g. due to previous adverse reactions to contrast media, severe claustrophobia or medical implants incompatible with MRI. In these patients mammography, ultrasound or CBE may have to be performed at a higher frequency, or in case of BRCA1/2 mutation carriers risk-reducing surgeries may play a bigger role.

\section{Mammography}

Mammography represents the cornerstone of breast cancer screening in the general population, especially in the age group $50-70$. It is the only breast screening modality for which a significant reduction in breast cancer mortality has been shown in large randomized trials $[32,33]$. In several countries, highly efficient and quality-assured mammography screening programs are in operation, which are able to offer this service to all women in the eligible age group. This capability is unique to mammography, since both ultrasound and breast MRI are too time consuming and expensive to be offered to the general population. However, mammography has several drawbacks. Sensitivity of mammography is relatively low, especially in women with dense breasts. It is accepted that up to $30 \%$ of the underlying (expected) breast cancer incidence rate in a screened population will be found as interval cancers in the first year after a screening mammogram, and up to $50 \%$ in the second year after screening [34]. Some of these cancers might have been detectable at the time of screening by other means. The risk associated with radiation exposure from modern, especially digital, mammography is small relative to the benefits in women 40 years and older [35]. However, the risk of radiation-induced breast cancer is higher in younger women, especially those younger than 30 [3]. There is also some concern that BRCA1/2 carriers may be at higher risk for radiation injury [36] since both genes are involved in the DNA repair mechanism. Evidence in this regard has so far been mixed [37-39], but at least one study has shown a slightly elevated risk of breast cancer in BRCA1/2 carriers exposed to radiation from diagnostic procedures before the age of 30 [40]. When high-risk screening programs were first set up more than 10 years ago, consensus based on expert opinion was to include strict annual mammography in the surveillance starting between age 25 and 35 for all high-risk patients [41-45]. However, with the improving technical quality of breast MRI and increasing reader experience, it has become evident that the additional absolute benefit of including mammography along with breast MRI in high-risk screening is small $[5,17,46]$, especially in high-risk women below the age of 40 , for whom even in BRCA1/2 mutation carriers the incidence of breast cancer is still relatively low. Therefore, in 2013 the GC-HBOC adjusted its high-risk surveillance program (table 1). Mammography is no longer recommended routinely for high-risk women below the age of 40 without a personal history of breast cancer if a high-quality breast MRI with little nonspecific background enhancement is available. A similar approach has been chosen for the recently set-up high-risk surveillance program in the UK (NHS Breast Screening Programme, NHSBSP), in which MRI only is offered as a screening tool in high-risk women below the age of $40[9,26]$. However, if MRI cannot be performed due existing contraindications or is limited due to very strong background enhancement, mammography will continue to play a role even in high-risk women under the age of 40 .

If possible, digital mammography rather than conventional film-screen mammography should be employed for high-risk screening [5]. Digital mammography has a higher sensitivity in women with dense breasts, which will be particularly beneficial, when screening young premenopausal high-risk women [47]. In addition, digital mammography has been found to be superior to conventional film-screen mammography in the detection of microcalcifications [48], the key feature of cancers detected solely by mammography, and not with ultrasound or MRI. The role of digital breast tomosynthesis (DBT), a new tomographic breast imaging technique based on a series of low-dose mammographic images obtained from different angles [49], is less clear for high-risk screening. This technique certainly plays an important role in the work-up of suspicious lesions seen primarily on MRI. Many of these lesions, even if not seen on the overview 2-dimensional (2D) 
Table 1. High-risk surveillance program with MRI of the GC-HBOC

\begin{tabular}{|c|c|}
\hline Established & 2005, recommendations updated in 2013 \\
\hline Screening centers & $\begin{array}{l}\text { currently } 15 \text { specialized university centers through- } \\
\text { out Germany, which also provide genetic } \\
\text { counseling and testing for high-risk women }\end{array}$ \\
\hline Funding & $\begin{array}{l}\text { financed through special integrated health care con- } \\
\text { tracts between payers and participating centers }\end{array}$ \\
\hline Entrance criteria & $\begin{array}{l}\text { carriers of a known mutation in a breast cancer } \\
\text { susceptibility gene or with high risk based on family } \\
\text { historya }\end{array}$ \\
\hline \multicolumn{2}{|l|}{ Covered age range, years } \\
\hline BRCA $1 / 2$ mutation carriersb & $25-70$ \\
\hline Carriers of other moderate risk genesd & $30-70 c$ \\
\hline High-risk women without a known mutation & $30-50$ \\
\hline MRI & annually \\
\hline \multicolumn{2}{|l|}{ Ultrasounde } \\
\hline BRCA1/2 mutation carriersb & every 6 months \\
\hline All others & annually \\
\hline Mammography & $\begin{array}{l}\text { individualized decision, e.g. every } 1-2 \text { years starting } \\
\text { at age } 40^{\mathrm{f}}\end{array}$ \\
\hline \multicolumn{2}{|c|}{$\begin{array}{l}\text { MRI = magnetic resonance imaging, GC-HBOC = German Consortium for Hereditary Breast and Ovarian } \\
\text { Cancer. }\end{array}$} \\
\hline \multicolumn{2}{|c|}{ probability of at least $20 \%$ based on Cyrillic 2.1 (http://www.cyrillicsoftware.com/). } \\
\hline \multicolumn{2}{|c|}{ 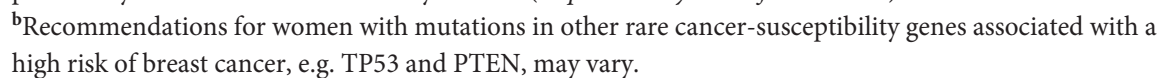 } \\
\hline \multirow{2}{*}{\multicolumn{2}{|c|}{$\begin{array}{l}\text { 'High-risk surveillance with MRI may be terminated before } \\
\text { mammographic density, but not before age } 50 \text {. }\end{array}$}} \\
\hline & \\
\hline \multicolumn{2}{|l|}{${ }^{\mathrm{d}}$ For example, RAD51C, RAD51D, CHEK2, PALB2. } \\
\hline \multicolumn{2}{|c|}{$\begin{array}{l}\text { eUltrasound is combined with a short clinical breast exam, history taking and if necessary counseling, e.g. } \\
\text { regarding other preventive measures. }\end{array}$} \\
\hline \multicolumn{2}{|c|}{$\begin{array}{l}\text { f Mammography before the age of } 40 \text { may be performed, e.g. for the following reasons: prior history of } \\
\text { breast cancer; MRI contraindicated or limited due to very strong background enhancement; further } \\
\text { work-up of suspicious findings on MRI, ultrasound or clinical breast exam. }\end{array}$} \\
\hline
\end{tabular}

mammogram, have some correlating finding on the tomosynthesis slices, allowing for exact 3D localization and if necessary facilitating biopsy. It has also been shown that the use of DBT in screening leads to a higher sensitivity, at least when DBT is used in addition to $2 \mathrm{D}$ mammography [50-52]. However, no studies are yet available to demonstrate what the additional benefit will be if DBT is used alongside MRI and/or ultrasound in a high-risk screening program. The benefit of DBT over 2D mammography is primarily related to non-calcified lesions, which are more likely to be visible on MRI and/or ultrasound [53]. Depiction of microcalcifications may in fact even be slightly inferior on DBT relative to digital 2D mammography [54]. DBT is, therefore, occasionally performed in combination with an additional 2D mammography, which would be inappropriate in high-risk women due to the associate increase in radiation exposure.

\section{Breast Ultrasound}

Breast ultrasound is a safe and easily performed procedure with virtually no contraindications. Whereas hand-held breast ultrasound examination performed by a physician, usually as an exten- sion of a CBE, is readily available in many European and Asian countries, use of breast ultrasound as part of breast cancer screening has traditionally been rare in the UK and North America. This has changed somewhat with the recent 'Are you dense' campaign in the US, requiring physicians in many states of the US to offer supplementary breast ultrasound in addition to mammography to women with dense breasts $[55,56]$. Even though the additional contribution of breast ultrasound performed alongside MRI in a high-risk screening program to breast cancer detection will be very low $[5,56]$, breast ultrasound can play an important role in highrisk screening. In the GC-HBOC, a short hand-held breast ultrasound in combination with a $\mathrm{CBE}$ is routinely performed by a physician preferably after and in full knowledge of the MRI (table 1). This allows for direct correlation of the MRI findings with the ultrasound and the clinical exam. Many nonspecific enhancing lesions on MRI, such as fibroadenomas or lymph nodes, can easily be identified on targeted ultrasound and thus the specificity of MRI can be increased [57]. Short-term follow-up, and if necessary biopsy, can then safely be performed by ultrasound. This also obviates the need for a formal recall system with its associated anxiety for the patients, since MRI findings can be discussed with all women in person. This is especially relevant when considering that 
Fig. 1. High-risk female patient with a known pathogenic mutation in the BRCA1 gene, who entered the intensified breast surveillance program of the German Consortium for Hereditary Breast and Ovarian Cancer (GC-HBOC) at the Charité in Berlin, Germany, in 2005 at age 31 without a prior history of cancer. In 2011 at age 37 a new 9-mm hypoechoic lesion was found in the upper-outer quadrant of the left breast on a regular scheduled 6-month interval ultrasound exam (a). Except for a slightly microlobulated margin the lesion exhibited predominantly benign ultrasound features such as oval shape, parallel orientation of the long axis, and posterior enhancement. A targeted mammographic view (b) with a metallic marker placed on the skin over the ultrasound lesion showed a few isolated non-suspicious calcifications as well as a very vague density in the area of the tumor. Repeat magnetic resonance imaging (MRI) (c) performed because of the abnormal ultrasound finding showed a corresponding subtle enhancing mass in the left breast (arrow) with typical ring enhancement suspicious for a high-grade malignancy. This lesion was not present even in retrospect on the last prior regular annual screening MRI exam 6 months earlier. Ultrasound-guided core biopsy confirmed a triple-negative breast cancer (TNBC) with a proliferation rate (Ki-67) of more than 90\%. Sentinel-node biopsy performed before initiation of neoadjuvant chemotherapy was negative ( $\mathrm{pN} 0(\mathrm{sn})(0 / 4))$ and the patient had pathological complete remission ( $\mathrm{pCR}$ ) at breast-conserving surgery following completion of neoadjuvant chemotherapy with carboplatin and docetaxel. In 2012 at age 38, after completion of adjuvant radiation therapy, the patient underwent risk-reducing salpingo-oophorectomy as recommended by the GC-HBOC for BRCA1/2 carriers around age 40. She resumed regular high-risk breast surveillance in 2012 and is currently healthy without evidence of recurrence or metastatic disease 3.5 years after her breast cancer diagnosis.

in an organized high-risk multimodal screening program the total recall rate of MRI and mammography combined can be as high as $25 \%$, at least in the first prevalent round [5]. The patient contact time during the ultrasound exam can be used for obtaining a short history as well as counseling, e.g. regarding other risk-reducing measures or associated non-breast cancer risks. Offering breast ultrasound and CBE as part of a high-risk surveillance program is usually well accepted and considered reassuring by the participating women [20]. In the GC-HBOC, ultrasound is offered in the 6-month interval between the annual MRI exams to women with a known mutation in one of the high-penetrance breast cancer susceptibility genes (table 1, fig. 1). In these cases the ultrasound at the time of the annual exam also serves as important comparison for the semiannual ultrasound-only exam. Breast ultrasound is the modality of choice during pregnancy and lactation, as both MRI and mammography are contraindicated during pregnancy and often severely limited during lactation. During this time, it may be reasonable to offer ultrasound in shorter intervals, e.g. every 3 months. An argument against the performance of breast ultrasound as part of screening is usually the high number of false-positive findings. However, if performed alongside MRI, many small nonspecific hypoechoic lesions on ultrasound can safely be ignored or subjected to short-term follow-up if no abnormal enhancement is seen on MRI in the region of the ultrasound abnormality.

Recently, new automated whole-breast ultrasound systems have been proposed as a substitute for hand-held ultrasound devices in screening [58]. However, the interpretation time for a full bilateral automated $3 \mathrm{D}$ breast ultrasound dataset is almost as long as the exam time for a tailored hand-held ultrasound by an experienced physician [59], and the automated ultrasound lacks the 2 key aspects that make ultrasound an important component of high-risk surveillance: the reassuring direct contact of the patient with a physician and the possibility to tailor the ultrasound exam specifically for the correlation of nonspecific MRI findings.

\section{Genotype-Phenotype Correlation and Implications for Screening}

Genetic changes in high-risk women do not only influence the frequency and age at which breast cancer is likely to occur [1], but also the histological type and imaging presentation of breast cancers differ depending on the underlying genetic abnormality [60, 61]. Knowledge of the genetic status of a particular patient is, 
therefore, crucial for choosing the optimal individualized surveillance strategy in high-risk patients. Fast-growing TNBCs are very common in BRCA1 mutation carriers, representing around $69 \%$ of all invasive cancers found in BRCA1 carriers. By contrast, only around $16 \%$ of all invasive cancers found in BRCA2 carriers are TNBCs. Interestingly, the share of triple-negative tumors seems to decrease with age for BRCA1 carriers, but to increase with age for BRCA2 carriers [60]. BRCA1-associated TNBCs often lack typical mammographic features of malignancy, such as spiculated margins or microcalcifications, and are therefore more often occult on mammography [62-65], decreasing the importance of mammography for the surveillance of BRCA1-positive patients [66] (fig. 1). On ultrasound, the majority of BRCA1-associated breast cancers with a size of more than $5 \mathrm{~mm}$ will be visible on targeted ultrasound as a hypoechoic solid mass, but may lack some of the typical sonographic features of malignancy, such as irregular shape, posterior acoustic shadowing or angulated/spiculated margins $[67,68]$ (fig. 1). Even though based on morphological criteria TNBCs may also appear benign on MRI, the correct diagnosis can usually be made due to typical malignant contrast enhancement features, such as rapid initial uptake or rim enhancement [69] (fig. 1). Unfortunately, several of the older studies on the imaging features of BRCA1-associated cancers did not differentiate between TNBCs and other cancers in BRCA1 carriers, although it can be assumed that the majority of the BRCA1-associated cancers in these studies would have been TNBCs. Imaging findings in BRCA2-associated breast cancers are much more similar to sporadic breast cancers, including a significantly higher proportion of in situ cancers with typical microcalcifications on mammography [61]. Another example of an association between immunohistological tumor properties and imaging features is the observation that estrogen receptornegative and HER2-positive cancers, which are rare in both BRCA1 and BRCA2 carriers [70], are often associated with typical malignant mammographic features, such as spiculated margins and pleomorphic microcalcifications [63].

\section{Impact on Mortality}

In women with a genetic predisposition for breast cancer, anxiety regarding breast or ovarian cancer mortality is very common, and is a major motivation to seek counseling and to participate in prevention measures. Many women have witnessed close relatives die of breast or ovarian cancer and they are determined to prevent the same fate for themselves. Based on model calculations, it is estimated that without intervention, $47 \%$ of BRCA1 mutation carriers und $29 \%$ of BRCA2 mutation carriers will die before reaching age 70 , compared to $16 \%$ in the general US population [71]. In this situation, offering early detection measures for secondary prevention to high-risk women means taking on a large responsibility, especially as effective surgical prevention measures such as bilateral risk-reducing mastectomy and/or salpingo-oophorectomy are available $[72,73]$. Unfortunately, very few reliable data are available regarding the effectiveness of intensified surveillance in high- risk women in regards to reducing breast cancer mortality $[6,22$, 74]. No randomized trials are available and are unlikely to occur due to the ethical dilemma of having to offer no screening to one arm of the trial. In addition, comparison with historical cohorts is difficult due to the substantial advances in targeted treatment and an increasing frequency of risk-reducing surgeries in high-risk women. Several prospective observational studies have shown a very favorable stage distribution of cancers detected by intensified multimodal surveillance programs [17, 23, 61, 75-77], which can be used as surrogate parameter for the reduction in breast cancer mortality.

However, intensified surveillance appears to be somewhat less effective in BRCA1 mutation carriers [6], in whom more advanced stage cancers, symptomatic interval cancers as well as breast cancer deaths have been observed despite regular screening with MRI in several of the high-risk surveillance observational studies [61, 66, $73,78,79]$. In the analysis of Heijnsdijk et al. [61], which combined data from the Dutch, Canadian and UK high-risk screening trials, the share of $\mathrm{T} 1 \mathrm{c}$ and $\mathrm{T} 2+$ tumors was $29 \%$ and $22 \%$, respectively, for BRCA 1 mutation carriers, compared to $16 \%$ and $4 \%$ for BRCA2 mutation carriers. There are 2 other factors that may also contribute to a less favorable outcome for surveillance-only strategies in BRCA1 carriers. There is evidence that the strong correlation between tumor size at detection and prognosis usually observed in breast cancer patients is much weaker for basal-like TNBCs, which are frequently found in BRCA1 mutation carriers [80]. A substantial portion of these cancers will have or will develop metastatic disease even if found at a size of less than $10-15 \mathrm{~mm}$, and prognosis is less favorable than for other types of breast cancers found at the same size $[6,81]$. In addition, due to the relatively poor prognosis and the lack of hormone receptors, the majority of TNBCs will require aggressive adjuvant chemotherapy, even if the cancer was found at a small size during intensified surveillance [21]. These factors contribute to the fact that prophylactic mastectomy (both primary and secondary) is now more actively offered to BRCA1 mutation carriers, and uptake of prophylactic mastectomy as riskreducing surgery has been increasing in this patient group in recent years. However, even in women who eventually opt for riskreducing bilateral mastectomy, intensified surveillance may be helpful as a temporary measure to delay the decision, e.g. until after completion of family planning.

When evaluating the efficiency of high-risk screening, it has to be borne in mind that both survival times and relative stage distribution of observed cancers during screening [82] will be influenced by a likely substantial detection lead-time for non-TNBCs through aggressive multimodal screening with MRI. On the other hand, the issue of overdiagnosis, which must be considered in populationbased mammography screening of postmenopausal women [33], will likely be of minor importance in screening young high-risk patients. The potentially long lead time of MRI-based screening in combination with the prolonged natural history of breast cancer also means that the mortality evaluation in high risk screening must include very long and complete follow-up for 10 years or more. This will be difficult to achieve for young mobile women of 
child-bearing age without accurate nationwide cancer registry linkage, as done in the recent Norwegian trial of BRCA1 mutation carriers undergoing high-risk surveillance with MRI as part of a national screening program [6].

\section{Conclusion}

Imaging-based surveillance for early detection of breast cancer is an important component of the care for high-risk women along with other preventive measures. Multimodality screening with inclusion of MRI can detect the vast majority of breast cancers at an early, potentially curable stage. However, for an adequate assessment of the safety of this approach, further long-term follow-up of high-risk women undergoing intensified breast surveillance is urgently needed, especially as other risk-reducing surgical measures are available and have been demonstrated to be highly effective in reducing breast cancer mortality in high-risk women.

\section{Disclosure Statement}

The author is spokesperson of the Center for Hereditary Breast and Ovarian Cancer in Berlin, Germany, and acts as reference radiologist in the GC-HBOC.

\section{References}

1 Mavaddat N, Peock S, Frost D, et al.: Cancer risks for BRCA1 and BRCA2 mutation carriers: Results from prospective analysis of EMBRACE. J Natl Cancer Inst 2013;105:812-822.

2 Carney PA, Miglioretti DL, Yankaskas BC, et al.: Individual and combined effects of age, breast density, and hormone replacement therapy use on the accuracy of screening mammography. Ann Intern Med 2003;138: 168-175.

3 Law J, Faulkner K: Cancers detected and induced, and associated risk and benefit, in a breast screening programme. Br J Radiol 2001;74:1121-1127.

4 Sardanelli F, Boetes C, Borisch B, et al.: Magnetic resonance imaging of the breast: Recommendations from the EUSOMA working group. Eur J Cancer 2010;46: 1296-1316.

5 Chiarelli AM, Prummel MV, Muradali D, et al.: Effectiveness of screening with annual magnetic resonance imaging and mammography: Results of the initial screen from the Ontario High Risk Breast Screening Program. J Clin Oncol 2014;32:2224-2230.

6 Moller P, Stormorken A, Jonsrud C, et al.: Survival of patients with BRCAl-associated breast cancer diagnosed in an MRI-based surveillance program. Breast Cancer Res Treat 2013;139:155-161.

7 Nkondjock A, Robidoux A, Paredes Y, et al.: Diet, lifestyle and BRCA-related breast cancer risk among French-Canadians. Breast Cancer Res Treat 2006;98:285-294.

8 Richter S, Haroun I, Graham TC, et al.: Variants of unknown significance in BRCA testing: Impact on risk perception, worry, prevention and counseling. Ann Oncol 2013;24 Suppl 8:viii69-viii74.

9 National Institute for Health and Care Excellence: Familial breast cancer: Classification and care of people at risk of familial breast cancer and management of breast cancer and related risks in people with a family history of breast cancer. Update of clinical guideline 14 and 41. (Clinical guideline 164.) 2013. http://guidance.nice.org. uk/CG164.

10 Saslow D, Boetes C, Burke W, et al.: American Cancer Society guidelines for breast screening with MRI as an adjunct to mammography. CA Cancer J Clin 2007;57:75-89.

11 Meindl A, Ditsch N, Kast K, et al.: Hereditary breast and ovarian cancer: new genes, new treatments, new concepts. Dtsch Arztebl Int 2011;108:323-330.

12 Fischer C, Kuchenbacker K, Engel C, et al.: Evaluating the performance of the breast cancer genetic risk models BOADICEA, IBIS, BRCAPRO and Claus for predicting BRCA1/2 mutation carrier probabilities: A study based on 7352 families from the German Hereditary Breast and Ovarian Cancer Consortium. J Med Genet 2013;50:360-367.
13 Schellong G, Riepenhausen M, Ehlert K, et al.: Breast cancer in young women after treatment for Hodgkin's disease during childhood or adolescence-an observational study with up to 33-year follow-up. Dtsch Arztebl Int 2014;111:3-9.

14 Antoniou A, Pharoah PD, Narod S, et al.: Average risks of breast and ovarian cancer associated with BRCA1 or BRCA2 mutations detected in case series unselected for family history: A combined analysis of 22 studies. Am J Hum Genet 2003;72:1117-1130.

15 Kamihara J, Rana HQ, Garber JE: Germline TP53 mutations and the changing landscape of Li-Fraumeni syndrome. Hum Mutat 2014;35:654-662.

16 Saadatmand S, Vos JR, Hooning MJ, et al.: Relevance and efficacy of breast cancer screening in BRCA1 and BRCA2 mutation carriers above 60 years: A national cohort study. Int J Cancer 2014;135:2940-2949.

17 Passaperuma K, Warner E, Causer PA, et al.: Longterm results of screening with magnetic resonance imaging in women with BRCA mutations. Br J Cancer 2012;127:24-30

18 Baltzer HL, Alonzo-Proulx O, Mainprize JG, et al.: MRI volumetric analysis of breast fibroglandular tissue to assess risk of the spared nipple in BRCA1 and BRCA2 mutation carriers. Ann Surg Oncol 2014;21:1583-1588.

19 Tilanus-Linthorst MM, Obdeijn IM, Hop WC, et al.: BRCAl mutation and young age predict fast breast cancer growth in the Dutch, United Kingdom, and Canadian magnetic resonance imaging screening trials. Clin Cancer Res 2007;13:7357-7362.

20 Spiegel TN, Hill KA, Warner E: The attitudes of women with BRCA1 and BRCA2 mutations toward clinical breast examinations and breast self-examinations. J Womens Health (Larchmt) 2009;18:1019-1024.

21 Chereau E, Uzan C, Balleyguier C, et al.: Characteristics, treatment, and outcome of breast cancers diagnosed in BRCA1 and BRCA2 gene mutation carriers in intensive screening programs including magnetic resonance imaging. Clin Breast Cancer 2010;10:113-118.

22 Gareth ED, Nisha K, Yit L, et al.: MRI breast screening in high-risk women: Cancer detection and survival analysis. Breast Cancer Res Treat 2014;145:663-672.

23 Sardanelli F, Podo F, Santoro F, et al.: Multicenter surveillance of women at high genetic breast cancer risk using mammography, ultrasonography, and contrastenhanced magnetic resonance imaging (the High Breast Cancer Risk Italian 1 Study): Final results. Invest Radiol 2011;46:94-105.

24 Warner E, Causer PA, Wong JW, et al.: Improvement in DCIS detection rates by MRI over time in a high-risk breast screening study. Breast J 2011;17:9-17.
25 Obdeijn IM, Loo CE, Rijnsburger AJ, et al.: Assessment of false-negative cases of breast MR imaging in women with a familial or genetic predisposition. Breast Cancer Res Treat 2010;119:399-407.

26 Jenkins J, Sellars SJ, Wheatin M: Guidelines on organising the surveillance of women at higher risk of developing breast cancer in an NHS Breast Screening Programme. NHSBSP Publication No. 73. Sheffield: NHS Cancer Screening Programmes, 2013.

27 Clayton D, Davison C, Bailey C, et al.: Technical guidelines for magnetic resonance imaging (MRI) for the surveillance of women at higher risk of developing breast cancer. NHSBSP Publication Number 68. Sheffield: NHS Cancer Screening Programmes, 2012.

28 Kuhl CK, Bieling HB, Gieseke J, et al.: Healthy premenopausal breast parenchyma in dynamic contrast-enhanced MR imaging of the breast: normal contrast medium enhancement and cyclical-phase dependency. Radiology 1997;203:137-144

29 Giess CS, Raza S, Birdwell RL: Patterns of nonmasslike enhancement at screening breast MR IMAGING of high-risk premenopausal women. Radiographics 2013; 33:1343-1360.

30 Heil J, Czink E, Schipp A, et al.: Detected, yet not diagnosed - breast cancer screening with MRI mammography in high-risk women. Breast care 2012;7:236-239.

31 Hao D, Ai T, Goerner F, et al.: MRI contrast agents: Basic chemistry and safety. J Magn Reson Imaging 2012;36:1060-1071

32 Nelson HD, Tyne K, Naik A, et al.: Screening for breast cancer: an update for the U.S. Preventive Services Task Force. Ann Intern Med 2009;151:727-737.

33 Marmot MG, Altman DG, Cameron DA, et al.: The benefits and harms of breast cancer screening: An independent review. Br J Cancer 2013;108:2205-2240.

34 Perry N, Broeders M, de Wolf C, et al.: European guidelines for quality assurance in breast cancer screening and diagnosis. Luxembourg: Office for Official Publications of the European Communities, 2006.

35 Yaffe MJ, Mainprize JG: Risk of radiation-induced breast cancer from mammographic screening. Radiology 2011;258:98-105.

36 Speit G, Trenz K: Chromosomal mutagen sensitivity associated with mutations in BRCA genes. Cytogenet Genome Res 2004;104:325-332.

37 Bernstein JL, Thomas DC, Shore RE, et al.: Contralateral breast cancer after radiotherapy among BRCA1 and BRCA2 mutation carriers: A WECARE Study Report. Eur J Cancer 2013;49:2979-2985.

38 Frankenberg-Schwager M, Gregus A: Chromosomal instability induced by mammography X-rays in primary human fibroblasts from BRCA1 and BRCA2 mutation carriers. Int J Radiat Biol 2012;88:846-857. 
\$3 Jansen-van der Weide MC, Greuter MJ, Jansen L, et al.: Exposure to low-dose radiation and the risk of breas cancer among women with a familial or genetic predisposition: a meta-analysis. Eur Radiol 2010;20:2547-2556.

40 Pijpe A, Andrieu N, Easton DF, et al.: Exposure to diagnostic radiation and risk of breast cancer among carriers of BRCA1/2 mutations: Retrospective cohort study (GENE-RAD-RISK). BMJ 2012;345:e5660.

41 Bick U: An integrated early detection concept in women with a genetic predisposition for breast cancer. Radiologe 1997;37:591-596.

42 Burke W, Daly M, Garber J, et al.: Recommendations for follow-up care of individuals with an inherited predisposition to cancer. II. BRCA1 and BRCA2. Cancer Genetics Studies Consortium. JAMA 1997;277:997-1003.

43 Warner E, Plewes DB, Hill KA, et al.: Surveillance of BRCA1 and BRCA2 mutation carriers with magnetic resonance imaging, ultrasound, mammography, and clinical breast examination. JAMA 2004;292:1317-1325.

44 Leach MO, Boggis CR, Dixon AK, et al.: Screening with magnetic resonance imaging and mammography of a UK population at high familial risk of breast cancer: A prospective multicentre cohort study (MARIBS). Lancet 2005;365:1769-1778

45 Kriege M, Brekelmans CT, Boetes C, et al.: Efficacy of MRI and mammography for breast-cancer screening in women with a familial or genetic predisposition. $\mathrm{N}$ Engl J Med 2004;351:427-437.

46 Kuhl C, Weigel S, Schrading S, et al.: Prospective multicenter cohort study to refine management recommendations for women at elevated familial risk of breast cancer: The EVA trial. J Clin Oncol 2010;28:1450-1457.

47 Pisano ED, Gatsonis C, Hendrick E, et al.: Diagnostic performance of digital versus film mammography for breast-cancer screening. N Engl J Med 2005;353:17731783 .

48 Bluekens AM, Holland R, Karssemeijer N, et al.: Comparison of digital screening mammography and screenfilm mammography in the early detection of clinically relevant cancers: a multicenter study. Radiology 2012; 265:707-714.

49 Baker JA, Lo JY: Breast tomosynthesis: State-of-the-art and review of the literature. Acad Radiol 2011;18:12981310 .

50 Skaane P, Bandos AI, Gullien R, et al.: Comparison of digital mammography alone and digital mammography plus tomosynthesis in a population-based screening program. Radiology 2013;267:47-56.

51 Houssami N, Skaane P: Overview of the evidence on digital breast tomosynthesis in breast cancer detection. Breast 2013;22:101-108

52 Ciatto S, Houssami N, Bernardi D, et al.: Integration of 3D digital mammography with tomosynthesis for population breast-cancer screening (STORM): A prospective comparison study. Lancet Oncol 2013;14:583-589.

53 Teertstra HJ, Loo CE, van den Bosch MA, et al.: Breast tomosynthesis in clinical practice: initial results. Eur Radiol 2010;20:16-24.

54 Spangler ML, Zuley ML, Sumkin JH, et al.: Detection and classification of calcifications on digital breast tomosynthesis and 2D digital mammography: a comparison. AJR 2011;196:320-324.
5 Cappello NM: Decade of 'normal' mammography reports-the happygram. J Am Coll Radiol 2013;10:903908

56 Berg WA, Mendelson EB: Technologist-performed handheld screening breast US imaging: How is it performed and what are the outcomes to date? Radiology 2014;272:12-27.

57 Sim LSJ, Hendriks J, Bult P, et al.: US correlation for MRI-detected breast lesions in women with familial risk of breast cancer. Clin Radiol 2005;60:801-806.

58 Giuliano V, Giuliano C: Improved breast cancer detection in asymptomatic women using $3 \mathrm{D}$-automated breast ultrasound in mammographically dense breasts. Clin Imaging 2013;37:480-486.

59 Prosch H, Halbwachs C, Strobl C, et al.: Automated breast ultrasound vs. handheld ultrasound: BI-RADS classification, duration of the examination and patient comfort. Ultraschall Med 2011;32:504-510.

60 Mavaddat N, Barrowdale D, Andrulis IL, et al.: Pathology of breast and ovarian cancers among BRCA1 and BRCA2 mutation carriers: Results from the Consortium of Investigators of Modifiers of BRCA1/2 (CIMBA). Cancer Epidemiol Biomarkers Prev 2012;21:134-147.

61 Heijnsdijk EAM, Warner E, Gilbert FJ, et al.: Differences in natural history between breast cancers in BRCA 1 and BRCA2 mutation carriers and effects of MRI Screening-MRISC, MARIBS, and Canadian studies combined. Cancer Epidemiol Biomarkers Prev 2012;21:1458-1468

62 Tilanus-Linthorst M, Verhoog L, Obdeijn IM, et al.: A BRCA1/2 mutation, high breast density and prominent pushing margins of a tumor independently contribute to a frequent false-negative mammography. Int J Cancer 2002;102:91-95.

63 Wang YB, Ikeda DM, Narasimhan B, et al.: Estrogen receptor-negative invasive breast cancer: Imaging features of tumors with and without human epidermal growth factor receptor type 2 overexpression. Radiology 2008;246:367-375.

64 Kaas R, Kroger R, Hendriks J, et al.: The significance of circumscribed malignant mammographic masses in the surveillance of BRCA 1/2 gene mutation carriers. Eur Radiol 2004;14:1647-1653.

65 Schrading S, Kuhl CK: Mammographic, US, and MR imaging phenotypes of familial breast cancer. Radiology 2008;246:58-70.

66 Obdeijn IM, Winter-Warnars GAO, Mann RM, et al.: Should we screen BRCA1 mutation carriers only with MRI? A multicenter study. Breast Cancer Res Treat 2014;144:577-582

67 Mesurolle B, Kadoch L, El-Khoury M, et al.: Sonographic features of breast carcinoma presenting as masses in BRCA gene mutation carriers. J Ultrasound Med 2007;26:817-824.

68 Rhiem K, Flucke U, Schmutzler RK: BRCA1-associated breast carcinomas frequently present with benign sonographic features. AJR Am J Roentgenol 2006; 186:E11-E12.

69 Uematsu T, Kasami M, Yuen S: Triple-negative breast cancer: Correlation between MR imaging and pathologic findings. Radiology 2009;250:638-647.
70 Lakhani SR, van de Vijver MJ, Jacquemier J, et al.: The pathology of familial breast cancer: Predictive value of immunohistochemical markers estrogen receptor, progesterone receptor, HER-2, and p53 in patients with mutations in BRCA1 and BRCA2. J Clin Oncol 2002; 20:2310-2318.

71 Kurian AW, Sigal BM, Plevritis SK: Survival analysis of cancer risk reduction strategies for BRCA1/2 mutation carriers. J Clin Oncol 2010;28:222-231.

72 Chai X, Friebel TM, Singer CF, et al.: Use of risk-reducing surgeries in a prospective cohort of 1,499 BRCA1 and BRCA2 mutation carriers. Breast Cancer Res Treat 2014;148:397-406.

73 Heemskerk-Gerritsen BA, Menke-Pluijmers MB, Jager A, et al.: Substantial breast cancer risk reduction and potential survival benefit after bilateral mastectomy when compared with surveillance in healthy BRCA1 and BRCA2 mutation carriers: A prospective analysis. Ann Oncol 2013;24:2029-2035.

74 Nelson HD, Pappas M, Zakher B, et al.: Risk assessment, genetic counseling, and genetic testing for BRCA-related cancer in women: A systematic review to update the U.S. Preventive Services Task Force Recommendation. Ann Intern Med 2014;160:255-266.

75 Warner E, Messersmith H, Causer P, et al.: Systematic review: Using magnetic resonance imaging to screen women at high risk for breast cancer. Ann Intern Med 2008;148:671-679.

76 Warner E, Hill K, Causer P, et al.: Prospective study of breast cancer incidence in women with a BRCAl or BRCA2 mutation under surveillance with and without magnetic resonance imaging. J Clin Oncol 2011;29: 1664-1669.

77 Leach MO: Breast cancer screening in women at high risk using MRI. NMR Biomed 2009;22:17-27.

78 Moller P, Evans DG, Reis MM, et al.: Surveillance for familial breast cancer: Differences in outcome according to BRCA mutation status. Int J Cancer 2007;121: 1017-1020.

79 Rijnsburger AJ, Obdeijn IM, Kaas R, et al.: BRCA1-associated breast cancers present differently from BRCA2-associated and familial cases: Long-term follow-up of the Dutch MRISC Screening Study. J Clin Oncol 2010;28:5265-5273.

80 Foulkes WD, Grainge MJ, Rakha EA, et al.: Tumor size is an unreliable predictor of prognosis in basal-like breast cancers and does not correlate closely with lymph node status. Breast Cancer Res Treat 2009;117: 199-204.

81 Evans AJ, Rakha EA, Pinder SE, et al.: Basal phenotype: a powerful prognostic factor in small screen-detected invasive breast cancer with long-term follow-up. J Med Screen 2007; 14:210-214.

82 Shen Y, Yang Y, Inoue LY, et al.: Role of detection method in predicting breast cancer survival: analysis of randomized screening trials. J Natl Cancer Inst 2005; 97:1195-1203. 